\title{
PRECIPITAÇÃO MÁXIMA DIÁRIA ANUAL E ESTIMATIVA DA EQUAÇÃO DE CHUVAS INTENSAS DO MUNICÍPIO DE DIVINÓPOLIS, MG, BRASIL
}

Annual daily maximum rainfall and estimate of intense rain equation for the Divinópolis municipality, MG, Brazil

Carolina Teixeira da Silva Miranda ${ }^{1 *}$; Michael Silveira Thebaldi2 ${ }^{*}$; Giovana Mara Rodrigues Borges Rocha ${ }^{3}$

\begin{abstract}
${ }^{1}$ Bacharela em Engenharia Ambiental e Sanitária; Centro Universitário de Formiga - MG; email: carolzinhatmiranda.cm@gmail.com 2Doutor em Recursos Hídricos em Sistemas Agrícolas, Centro Universitário de Formiga - MG; msthebaldi@uniformg.edu.br *Autor para correspondência

${ }_{3}^{3}$ Bacharela em Engenharia Ambiental e Sanitária; Centro Universitário de Formiga - MG; giovanaengambiental@gmail.com
\end{abstract}

Artigo enviado em 21/12/2016, aceito em 28/11/2017 e publicado em 20/12/2017.

\begin{abstract}
Resumo - A precipitação representa a principal entrada de água do ciclo hidrológico, sendo fundamental para o entendimento do balanço hídrico, assim, conhecer o comportamento probabilístico de dados de precipitação é extremamente importante para a elaboração de estudos estratégicos associados ao planejamento do meio ambiente, tanto em áreas urbanas, como rurais. Deste modo, este trabalho teve como objetivo estudar o comportamento de modelos probabilísticos na predição da precipitação máxima diária anual, bem como modelos de chuvas intensas para o município de Divinópolis - MG. Para tal, os dados de precipitação máxima diária anual foram ordenados de forma decrescente, com o objetivo de identificar uma distribuição estatística que melhor a descrevesse por meio da frequência de excedência. Quanto ao estudo de chuvas intensas, foi utilizada a metodologia de desagregação de chuvas, sendo ajustados os modelos Intensidade-Duração-Frequência (IDF) e exponencial. Por meio do teste do Qui-Quadrado, foi identificado que o modelo que representou melhor os dados de precipitação máxima diária anual foi Gumbel para Máximos, enquanto para a equação de chuvas intensas, o modelo IDF foi o que melhor se ajustou à série de intensidades de precipitação.
\end{abstract}

Palavras-chave - ciclo hidrológico, gestão dos recursos hídricos, distribuições estatísticas.

Abstract - Rainfall is the main water inlet of the hydrological cycle, being central to the understanding of water balance. Thus, the knowledge of the probabilistic behavior of rainfall data is extremely important for the development of strategic studies associated with the planning of the environment at urban and agricultural areas. This work had as objective to study the behavior of probabilistic models to predict the annual maximum daily rainfall, as well heavy rain models for the city of Divinópolis - MG. To do this, annual maximum daily rainfall data were ranked in decreasing order that best describes the statistical distribution by exceedance probability. Daily rainfall disaggregation methodology was used for the intense rain model studies and adjusted with Intensity-Duration-Frequency (IDF) and Exponential models. By the use of chi-square test, it was identified that the model that best represents the maximum annual daily rainfall data was Gumbel, while for the equation of heavy rains, the IDF model was the best fit to the range of precipitation intensities.

Keywords - hydrological cycle, water resources management, statistical distributions.

\section{INTRODUÇÃO}

A precipitação pode ser definida como toda água que provém do meio atmosférico e atinge a superfície terrestre, sendo as chuvas um tipo de precipitação. Neste sentido, as chuvas intensas ou extremas, também comumente conhecidas como chuvas máximas, são caracterizadas por ter distribuição irregular tanto temporalmente, quanto espacialmente (ARAÚJO et al., 2008). Estas, apresentam grande lâmina precipitada, que supera um valor mínimo, em função de um intervalo de tempo (SILVA et al., 2003).
Segundo Mello et al. (2008), a análise de chuvas intensas baseia-se em um dos produtos mais aplicados da hidrologia. A análise de seus impactos é de crucial importância para a sociedade, uma vez que identifica áreas de risco para ocupação humana, dentre vários outros aspectos elementares ao saneamento básico (MELLO; VIOLA, 2013).

Dentre as ocorrências desencadeadas por chuvas intensas destacam-se as enchentes e inundações, que são termos frequentemente empregados como sinônimos, no entanto devem ser usados com diferenciação (KOBIYAMA et al., 2006). 
É indispensável dispor de informações relacionadas às variáveis climáticas no planejamento do uso da água e do solo, tal como as chuvas intensas. A caracterização da variabilidade temporal das chuvas intensas ao longo de sua duração é fundamental para quantificar adequadamente os efeitos acarretados no escoamento superficial em áreas urbanas e rurais (SILVA; CLARKE, 2004), sendo utilizada em estudos pertinentes ao dimensionamento de projetos hidráulicos, como obras para o controle em áreas agrícolas e urbanas e o armazenamento e fornecimento de água, tanto para irrigação, indústria, abastecimento doméstico, como para dessedentação animal (RODRIGUES et al. 2008; MORUZZI; OLIVEIRA 2009).

A caracterização das chuvas intensas é fundamental para se quantificar acertadamente seus efeitos. O interessante não é meramente quantificar o efeito de um evento hidrológico que acaba de ocorrer, mas, sobretudo, a capacidade de prever a ocorrência de eventos extremos e suas consequências, da forma mais precisa e real possível (CRUCIANI et al., 2002).

As séries de dados na análise de frequência de chuvas intensas podem ser anuais, quando constituídas pelos maiores valores observados em cada ano, parciais, quando compostas pelos maiores valores verificados no período total de observação, ou completa, quando formada por todos os valores disponíveis no estudo (FREITAS et al., 2001).

De acordo com Mello e Silva (2013), a alternativa para se gerar informações para chuvas intensas quando não se dispõe de pluviogramas é a aplicação de relações médias entre lâminas precipitadas em diferentes tempos. Com base nesse princípio, denominado desagregação de chuvas, as lâminas e intensidades de precipitação podem ser estimadas a partir da chuva máxima diária anual, registrada pelo pluviômetro, em intervalos de tempo tão pequenos quanto cinco minutos.

Assim, o objetivo deste trabalho foi estudar o comportamento de distribuições de frequências estatísticas na predição da precipitação máxima diária anual, bem como modelos de chuvas intensas para o município de Divinópolis, MG, Brasil.

\section{MATERIAL E MÉTODOS}

O município de Divinópolis está localizado no Oeste do estado de Minas Gerais e é constituído pelos biomas Cerrado e Mata Atlântica. O seu território do município possui uma área de $708,115 \mathrm{~km}^{2}$ e uma população estimada de 213.016 habitantes, conforme o último censo demográfico publicado no ano de 2010 (IBGE, 2016).

De acordo com a classificação climática de Köppen (ALVARES et al., 2013) o município se enquadra no clima Aw, ou seja, possui clima tropical, na qual no inverno existe muito menos pluviosidade que no verão e sua temperatura média é de $21,5^{\circ} \mathrm{C}$.

Os dados de precipitação do município de Divinópolis foram obtidos no serviço Hidroweb, controlado pela Agência Nacional das Águas (ANA). Foram utilizadas informações dos períodos entre 1942 e 2015, sendo que os anos de 1951, 1952, 1953, 1954, 1955, 1956, 1957, 1958, 1960, 1963, 1965, 1989, 1990, 1995, 1997 e 1998 não foram utilizados por não serem séries completas. Assim, foram considerados 58 anos de informações acerca da precipitação para o estudo.

\section{Precipitação Máxima Diária Anual}

Os dados foram ordenados com o propósito de se obter as precipitações máximas diárias anuais e subsequentemente organizados em ordem decrescente, a fim de se obter a frequência de excedência, constituindo uma série com 58 valores de precipitação máxima diária anual para Divinópolis - MG. As frequências observadas relativas aos eventos hidrológicos foram alcançadas através da fórmula de Weibull (Equação 1).

$\mathrm{f}_{\mathrm{obs}}=\frac{\mathrm{i}}{\mathrm{N}+1}$

em que:

$\mathrm{f}_{\mathrm{obs}}$ - frequência observada de excedência;

i - posição ocupada pelo dado dentro da série histórica; $\mathrm{N}$ - tamanho da série histórica.

Com base nos valores calculados da frequência de excedência, foram calculados os tempos de retorno das precipitações relacionados a cada frequência de excedência, como indicado na Equação 2.

$\mathrm{TR}=\frac{1}{\mathrm{f}_{\mathrm{obs}}}$

em que:

TR - tempo de retorno.

Ajuste das funções cumulativas de probabilidade

As funções cumulativas e densidade de probabilidade foram adaptadas nos modelos Gumbel para máximos, Fréchet, Gama, Log-Normal 2 parâmetros e LogNormal 3 parâmetros.

A distribuição Gumbel para máximos tem sua função densidade de probabilidade (FDP) representada pela equação 3 .

$\mathrm{f}(\mathrm{x})=\frac{1}{\alpha} \cdot \mathrm{e}^{\left[-\frac{(\mathrm{x}-\mu)}{\alpha}-\mathrm{e}^{-\frac{(\mathrm{x}-\mu)}{\alpha}}\right]}$

em que:

$\alpha$ e $\mu$ - são os parâmetros da distribuição.

A função cumulativa de probabilidade é dada pela Equação 4, em que x é o valor da variável hidrológica avaliada - no caso deste estudo precipitação máxima diária anual, e $\mathrm{x}_{\mathrm{i}}$ é um valor qualquer da mesma variável.

$P\left(x \geq x_{i}\right)=1-e^{-e^{-\alpha(x-\mu)}}$ 
Os parâmetros $\alpha$ e $\mu$ dados pelo método dos momentos são evidenciados nas equações 5 e 6 , e são presumidos a partir da média $(\bar{X})$ e desvio padrão (S) amostrais.

$\alpha=\frac{1,2826}{S}$

$\mu=\bar{X}-0,451 \cdot S$

A função de Fréchet fundamenta-se na aplicação da Distribuição Gumbel aos valores logaritmizados da variável hidrológica. Esta distribuição foi adotada para compreender as frequências de precipitações máximas. As funções densidade de probabilidade e cumulativa de probabilidade são representadas pelas equações 7 e 8 , nesta ordem.

$$
\begin{aligned}
& \mathrm{FDP}=\mathrm{f}(\mathrm{x})=\frac{\theta}{\lambda} \cdot\left(\frac{\lambda}{\mathrm{x}}\right)^{\theta+1} \cdot \exp \left[-\left(\frac{\lambda}{\mathrm{x}}\right)^{\theta}\right] \\
& \mathrm{P}(\mathrm{X} \geq \mathrm{xi})=1-\exp \left[-\left(\frac{\lambda}{\mathrm{x}}\right)^{\theta}\right]
\end{aligned}
$$

Sendo que, para $\mathrm{x}>0 ; \theta, \lambda>0$.

em que:

$\theta$ - parâmetro da distribuição associado à média;

$\lambda$ - parâmetro da distribuição associado à variância.

A obtenção dos parâmetros da distribuição Fréchet, $\theta$ e $\lambda$ se inicia com a Equação 9, a partir do coeficiente de variação (CV). Em seguida foi calculado o parâmetro $\lambda$ por meio das Equações 10 e 11, a partir do ajuste da média.

$\mathrm{CV}=\sqrt{\frac{\Gamma\left(1-\frac{2}{\theta}\right)}{\Gamma^{2}\left(1-\frac{1}{\theta}\right)}}-1$

$\mathrm{E}(\mathrm{x})=\overline{\mathrm{X}}=\lambda \cdot \Gamma\left(1-\frac{1}{\theta}\right) \operatorname{para} \theta>0$

$\operatorname{Var}(\mathrm{x})=\mathrm{s}^{2}=\lambda^{2} \cdot\left[\Gamma\left(1-\frac{2}{\theta}\right)-\Gamma^{2}\left(1-\frac{1}{\theta}\right)\right]$

Sendo $\theta>2$.

em que:

$\Gamma$ - função Gama.

A estimativa de um valor $\mathrm{x}$, vinculado a um TR, é dada através da equação 12.
$\mathrm{x}_{\mathrm{TR}}=\lambda \cdot\left[\mathrm{LN}\left(\frac{\mathrm{TR}}{\mathrm{TR}-1}\right)\right]^{-\frac{1}{\theta}}$

Já a função cumulativa de probabilidade da distribuição Gama é obtida pela equação 13.

$\mathrm{FCP}=\frac{1}{\beta^{v} \cdot \Gamma(v)} \int_{0}^{\mathrm{x}} \mathrm{u}^{(v-1)} \mathrm{e}^{\frac{-\mathrm{u}}{\beta}} \mathrm{du}$

Os parâmetros da distribuição Gama são dados pelas equações 14 e 15.

$\beta=\frac{S^{2}}{\bar{X}}$

$v=\frac{(\bar{X})^{2}}{S^{2}}$

em que:

$\mathrm{S}^{2}=$ desvio padrão amostral da série histórica;

$\overline{\mathrm{X}}=$ média amostral da série histórica.

A distribuição log-Normal 2 parâmetros é exposta pela função densidade de probabilidade como descrito na equação 16.

$f(x)=\frac{1}{X \cdot \sigma n \cdot \sqrt{2 \pi}} \cdot e^{-0.5\left(\frac{\operatorname{Ln}(x)-\mu n}{\sigma n}\right)^{2}}$

em que:

$\mu n$ e $\sigma$ n - parâmetros da distribuição, correspondendo à média e ao desvio padrão dos logaritmos dos dados.

A equação base para estudos hidrológicos, aplicando a distribuição log-Normal 2 parâmetros é adaptada da equação geral de Ven Te Chow (HAAN, 1979), conforme a equação 17.

$\mathrm{X}_{\mathrm{TR}}=\mathrm{e}^{\mu \mathrm{n}+\sigma \mathrm{n} \cdot \mathrm{K}_{\mathrm{TR}}}$

em que:

$\mathrm{X}_{\mathrm{TR}}$ - valor da variável hidrológica associada a um tempo de retorno;

$\mathrm{K}_{\mathrm{T}}$ - variável reduzida da distribuição Normal.

A distribuição log-Normal 3 parâmetros é apresentada pela função densidade de probabilidade, conforme descrito na Equação 18.

$f(x)=\frac{1}{(X-\beta) \cdot \sigma n \cdot \sqrt{2 \pi}} \cdot e^{-0.5\left(\frac{\operatorname{Ln}(x-\beta)-\mu n}{\sigma n}\right)^{2}}$

Sendo $x \geq \beta$.

Para a estimativa dos parâmetros da distribuição log-Normal 3 parâmetros foram utilizadas as equações 19 , $20,21,22$ e 23. 
$\varphi=\frac{\left[-\gamma+\left(\gamma^{2}+4\right)^{0,5}\right]}{2}$

$\eta y=\frac{\left(1-\varphi^{\frac{2}{3}}\right)}{\varphi^{\frac{1}{3}}}$

$\beta=\bar{X}-\frac{S}{\eta y}$

$\mu \mathrm{n}=\mathrm{LN}\left(\frac{\mathrm{S}}{\eta \mathrm{y}}\right)-0,5 \cdot \mathrm{LN}\left(\eta \mathrm{y}^{2}+1\right)$

$\sigma \mathrm{n}=\sqrt{\mathrm{LN}\left(\eta \mathrm{y}^{2}+1\right)}$

em que:

$\eta y$ - coeficiente de variação da variável $\mathrm{y}=\mathrm{x}-\beta$;

$\gamma$ - coeficiente de assimetria da série histórica.

A equação base para estudos hidrológicos, aplicando a distribuição log-Normal 3 parâmetros, também é adaptada da equação geral de Ven Te Chow (HAAN, 1979), conforme expresso na Equação 24.

$\mathrm{X}_{\mathrm{TR}}=\mathrm{e}^{\mu \mathrm{n}+\sigma \mathrm{n} \cdot \mathrm{K}_{\mathrm{TR}}}+\beta$

Com o objetivo de testar a aderência dos dados observados aos modelos referidos acima foram realizados os testes de Kolmogorov-Smirnov e Qui-Quadrado, levando em consideração um nível de significância de $5 \%$ de probabilidade estatística em ambos.

A seleção da distribuição estatística que representa adequadamente a série histórica de precipitação máxima diária anual foi realizada com base nos testes de aderência, sendo a distribuição selecionada a de representação significativa a $5 \%$ de probabilidade tanto para os testes de Kolmogorov-Smirnov e Qui-Quadrado e de menor valor de Qui-Quadrado calculado.

$\mathrm{Na}$ ocasião em que o teste de Qui-Quadrado não era significativo para nenhuma distribuição aplicada à série histórica de precipitação máxima diária anual, adotou-se como critério de escolha de distribuição aquela que fosse significativa pelo teste de Kolmogorov-Smirnov e apresentasse valor de Qui-Quadrado calculado mais próximo do Qui-Quadrado tabelado a significância de 5\% de probabilidade.

Já quando o teste de Kolmogorov-Smirnov não era significativo para nenhuma distribuição aplicada à série histórica de precipitação máxima diária anual, tomou-se como distribuição estatística a que melhor representaria a série, aquela significativa ao teste de Qui-Quadrado, possuindo menor valor de Qui-Quadrado calculado. Para séries históricas em que nenhuma distribuição possuísse aderência significativa à $5 \%$ de probabilidade em ambos os testes, foi feita seleção com base na distribuição com menor Qui-Quadrado calculado, mesmo que não houvesse confiança na representação da série pela distribuição.

\section{Desagregação de chuvas}

Com o intuito de se obter os valores das precipitações associados às durações, considerando o período de retorno de 5 a 500 anos e o tempo de duração de 5 a 1440 min, utilizou-se o método de desagregação de chuvas (FROEHLICH, 1993; CARDOSO et al., 1998). Associou-se, então, a precipitação de 1 dia, para um dado tempo de retorno e calculou-se a partir da distribuição estatística que melhor aderiu à série de precipitação máxima diária anual, com a precipitação de $24 \mathrm{~h}$ e, assim, sucessivamente, utilizando as constantes da Tabela 1.

Tabela 1. Constantes do modelo de desagregação de chuvas diárias

\begin{tabular}{cc}
\hline Relação (ht1/ht2) & Constante $(\mathrm{K})$ \\
\hline 1 dia $/ 24 \mathrm{~h}$ & 1,14 \\
$12 / 24 \mathrm{~h}$ & 0,85 \\
$10 \mathrm{~h} / 24 \mathrm{~h}$ & 0,82 \\
$8 / 24 \mathrm{~h}$ & 0,78 \\
$6 / 24 \mathrm{~h}$ & 0,72 \\
$1 / 24 \mathrm{~h}$ & 0,42 \\
$0,5 / 1 \mathrm{~h}$ & 0,74 \\
$25 \mathrm{~min} / 0,5 \mathrm{~h}$ & 0,91 \\
$20 \mathrm{~min} / 0,5 \mathrm{~h}$ & 0,81 \\
$15 \mathrm{~min} / 0,5 \mathrm{~h}$ & 0,70 \\
$10 \mathrm{~min} / 0,5 \mathrm{~h}$ & 0,54 \\
$5 \mathrm{~min} / 0,5 \mathrm{~h}$ & 0,34 \\
\hline
\end{tabular}

Fonte: Adaptado de Occhipinti e Santos (1966); Mello e Silva (2013).

Por definição, a precipitação de 1 dia refere-se àquela obtida por pluviômetro com intervalo de $24 \mathrm{~h}$ sempre fixo, enquanto a precipitação de $24 \mathrm{~h}$ é referente àquela obtida por pluviógrafo sem fixação do início da contagem do tempo (MELLO; SILVA, 2013).

Desta forma, dividindo a precipitação obtida pela sua respectiva duração, obteve-se as intensidades de precipitação associadas aos períodos de retorno e às durações. Determinou-se também as intensidades de precipitação consideradas como chuvas intensas, com base nos valores apresentados por Pinto (1995) e Mello e Silva (2013).

\section{Equação de chuvas intensas}

Os valores de intensidade de precipitação foram adaptados aos seguintes modelos:

- Equação IDF (modelo 1), como exposto na equação 26 :

$\mathrm{I}=\frac{\mathrm{K} \cdot \mathrm{TR}^{\mathrm{a}}}{(\mathrm{b}+\mathrm{td})^{\mathrm{c}}}$

em que:

I - intensidade de chuva $(\mathrm{mm} / \mathrm{h})$;

$\mathrm{K}$, a, b e c - parâmetros de ajuste;

TR - tempo de retorno (anos);

td - tempo de duração (minutos). 
- Modelo exponencial, apresentado conforme equação 27 :

$\mathrm{I}=\mathrm{e}^{\mathrm{B}+\mathrm{D} \cdot \mathrm{TR}^{\mathrm{x}}+\mathrm{E} \cdot(\ln \mathrm{td})^{2}}$

em que:

B, D, x e E - parâmetros de ajuste.

O critério determinante para a escolha do modelo exponencial foi a sua simplicidade, já que apresenta o mesmo número de parâmetros do modelo IDF. Landsberg (1977), enfatiza que os modelos estudados são empregados para modelagem de fenômenos em ciências naturais.

O ajuste dos modelos foi executado utilizando o pacote Solver do Microsoft Excel, utilizando o método GRG Não-Linear. Para a avaliação dos modelos, foram considerados o coeficiente de determinação ajustado $\left(\mathrm{R}^{2}\right)$ e o erro absoluto médio produzido pelos modelos em relação aos dados originais (PINTO, 1995), conforme equação 28.

$$
E A M=N^{-1} \sum_{i=1}^{N_{d}}\left|P_{i}-O_{i}\right|
$$

em que:

$\mathrm{N}$ - número de pares de dados;

$P_{i}$ - valor de intensidade de precipitação estimada pela equação de chuvas intensas (mm);

$\mathrm{O}_{\mathrm{i}}$ valor de intensidade de precipitação calculada através da desagregação de chuvas (mm).

\section{RESULTADOS E DISCUSSÃO}

Os valores máximo e mínimo identificados para a precipitação máxima diária anual foram de 149,3 mm e 35,1 $\mathrm{mm}$, respectivamente. Tem-se que a média dos valores de PMDA é de 78,44 mm, ao passo que o desvio padrão é de $21,79 \mathrm{~mm}$.

Já os resultados obtidos para os parâmetros de ajuste das distribuições estatísticas não-paramétricas avaliadas com os dados da série histórica de precipitação máxima diária anual do município de Divinópolis, são apresentados na Tabela 2 , enquanto que os valores de $\Delta \mathrm{F}$ calculado máximo e $\lambda^{2}$ calculado alcançados na avaliação da aderência dos modelos ajustados à distribuição de probabilidades da série histórica de precipitação máxima diária anual do município de Divinópolis - $M G$, são mostrados na Tabela 3.

Com exceção do modelo Fréchet, que não se ajustou aos dados observados de PMDA em ambos os testes de aderência realizados, todas as funções cumulativas de probabilidade tiveram aderência significativa, tanto pelo teste de Kolmogorov-Smirnov, quanto pelo Qui-Quadrado. Dentre os quatro métodos (Gumbel para máximos, Gama, Log-Normal 2P e Log-Normal 3P) que aderiram significativamente aos dados da série histórica, o melhor ajuste, com base no teste do Qui-Quadrado, se deu para o modelo Gumbel para Máximos, pois apresentou o menor valor de $\lambda^{2}$ calculado, 2,025 aproximadamente (Tabela 3).
Isto confirma os resultados encontrados na literatura sobre as distribuições de probabilidades aplicadas a valores de precipitação máxima diária anual, em que a distribuição de Gumbel para máximos surge como a mais indicada e melhor ajustada (NAGHETTINI; PINTO, 2007; SANSIGOLO, 2008).

Em comparação, Thebaldi (2012) realizou um estudo da precipitação máxima diária anual da cidade de Uberaba - MG e seus resultados corroboraram com os deste estudo, haja vista que as funções cumulativas de probabilidade (Gumbel para máximos, Gama, Log-Normal 2P e Log-Normal 3P) aderiram aos dados da série histórica, tanto pelo teste de Kolmogorov-Smirnov, quanto pelo QuiQuadrado e o melhor ajuste, assim como para Divinópolis, se deu para Gumbel para Máximos, que também apresentou o menor valor de $\lambda^{2}$ calculado.

Borges e Thebaldi (2016) ao avaliarem a precipitação máxima diária anual do município de Formiga - MG, observaram que o modelo de Gumbel para Máximos representou melhor os dados para esta localidade, assim, como ocorrido neste estudo.

Por meio da distribuição de Gumbel para máximos que apresentou melhor ajuste a série observada, foram calculados os valores de precipitações máximas diárias anuais, relativas a tempos de retorno de 5, 10, 20, 50, 100 e 500 anos. Estas foram submetidas à técnica de desagregação de chuvas, para indicação das lâminas precipitadas para tempos de duração de 5, 10, 15, 20, 25, 30, 60, 360, 480, 600, 720 e 1440 minutos, conforme apresentado na Tabela 4.

Analisando os dados, observa-se que o maior valor de precipitação máxima diária anual que é contemplada na série histórica $(149,3 \mathrm{~mm})$, só será igualada ou superada em um tempo de retorno de 500 anos quando relacionada a precipitação de um dia.

Além disso, tem-se que a maior lâmina precipitada calculada a partir do método de desagregação de chuvas, é relativa a um tempo de retorno de 500 anos e duração de 1440 minutos $(198,6 \mathrm{~mm})$, ao passo que a menor, está associada a um tempo de retorno de 5 anos e duração de 5 minutos $(11,3 \mathrm{~mm})$.

A série de intensidades de precipitação foi gerada a partir dos dados contidos na Tabela 4, e, ao ser ajustada aos modelos IDF e exponencial de chuvas intensas, constituiu os parâmetros de ajuste apresentados na Tabela 5. Nesta, tem-se que os modelos IDF e exponencial de chuvas intensas apresentaram bons ajustes com $\mathrm{R}^{2}$ superior a 0,99 em ambos os casos. Além disso, pelos valores obtidos do Erro Absoluto Médio, tem-se que o modelo IDF foi o mais eficiente ao representar as intensidades de precipitação calculadas a partir do método de desagregação de chuvas para o município estudado.

Mello et al. (2001) realizaram uma análise de modelos matemáticos aplicados ao estudo das chuvas intensas com dados provenientes da estação meteorológica da Universidade Federal de Lavras e os resultados obtidos corroboram com os deste estudo, sendo que os modelos não lineares (IDF e exponencial) geraram menores erros em comparação ao modelo linear, proporcionando melhores 
Tabela 2. Valores obtidos dos parâmetros de ajuste das funções avaliadas com os dados da série histórica de precipitação máxima diária anual do município de Divinópolis (MG).

\begin{tabular}{ccccc}
\hline Gumbel para máximos & Fréchet & Gama & Log-Normal 2P & Log-Normal 3P \\
\hline$\mu: 68,63759$ & $\lambda: 61,97208$ & $\beta: 6,052858$ & $\mu: 4,325758$ & $\beta:-0,72239$ \\
$\alpha: 0,058862$ & $\theta: 5,136216$ & v: 12,95968 & $\sigma: 0,273366$ & $\mu_{\mathrm{n}}: 4,335027$ \\
& & & & $\sigma_{\mathrm{n}}: 0,270237$ \\
\hline
\end{tabular}

Tabela 3. Valores obtidos das estatísticas dos testes de aderência de Kolmogorov-Smirnov e Qui-Quadrado na avaliação do ajuste das funções à série histórica de precipitação máxima diária anual do município de Divinópolis - MG.

\begin{tabular}{|c|c|c|c|c|}
\hline \multirow{2}{*}{ Modelo } & \multicolumn{4}{|c|}{ PMDA } \\
\hline & $\Delta \mathrm{F}$ calculado Máximo & $\Delta \mathrm{F}$ tabelado & $\lambda^{2}$ calculado & $\lambda^{2}$ tabelado \\
\hline Gumbel para máximos & $0,070269 *$ & & $2,024689439 *$ & \\
\hline Fréchet & $0,254403^{\mathrm{NS}}$ & & $10,64968801^{\mathrm{NS}}$ & \\
\hline Gama & $0,093415^{*}$ & 0,174 & $4,625222775^{*}$ & 7,815 \\
\hline Log-Normal 2P & $0,076938^{*}$ & & $3,187225648^{*}$ & \\
\hline Log-Normal 3P & $0,076443 *$ & & $3,202448627 *$ & \\
\hline
\end{tabular}

NS: não significativo à $5 \%$ de probabilidade; *Significativo a $5 \%$ de probabilidade

Tabela 4. Precipitação obtida pelo método da desagregação de chuvas, para o município de Divinópolis - MG.

\begin{tabular}{|c|c|c|c|c|c|c|c|c|c|c|c|c|c|}
\hline \multirow{3}{*}{ TR } & \multirow{3}{*}{ Pdia } & \multicolumn{12}{|c|}{ Tempo de duração (minutos) } \\
\hline & & 1440 & 720 & 600 & 480 & 360 & 60 & 30 & 25 & 20 & 15 & 10 & 5 \\
\hline & & \multicolumn{12}{|c|}{ Precipitação (mm) } \\
\hline 5 & 94,1 & 107,3 & 91,2 & 88,0 & 83,7 & 77,3 & 45,1 & 33,3 & 30,3 & 27,0 & 23,3 & 18,0 & 11,3 \\
\hline 10 & 106,9 & 121,8 & 103,6 & 99,9 & 95,0 & 87,7 & 51,2 & 37,9 & 34,5 & 30,7 & 26,5 & 20,4 & 12,9 \\
\hline 20 & 119,1 & 135,8 & 115,4 & 111,3 & 105,9 & 97,8 & 57,0 & 42,2 & 38,4 & 34,2 & 29,5 & 22,8 & 14,3 \\
\hline 50 & 134,9 & 153,8 & 130,7 & 126,1 & 120,0 & 110,7 & 64,6 & 47,8 & 43,5 & 38,7 & 33,5 & 25,8 & 16,3 \\
\hline 100 & 146,8 & 167,3 & 142,2 & 137,2 & 130,5 & 120,5 & 70,3 & 52,0 & 47,3 & 42,1 & 36,4 & 28,1 & 17,7 \\
\hline 500 & 174,2 & 198,6 & 168,8 & 162,8 & 154,9 & 143,0 & 83,4 & 61,7 & 56,2 & 50,0 & 43,2 & 33,3 & 21,0 \\
\hline
\end{tabular}

TR: tempo de retorno, em anos. Pdia: precipitação de um dia, em $\mathrm{mm}$.

Tabela 5. Parâmetros obtidos através dos ajustes dos modelos IDF e Exponencial aos dados de intensidade de precipitação obtidos pelo método da desagregação de chuvas.

\begin{tabular}{cccc}
\hline & Modelo IDF & \multicolumn{2}{c}{ Modelo Exponencial } \\
\hline $\mathrm{K}$ & 809,691961001355 & $\mathrm{~B}$ & 6,32964279035316 \\
$\mathrm{a}$ & 0,127576065684088 & $\mathrm{D}$ & $-1,56445699296022$ \\
$\mathrm{~b}$ & 9,78164971887621 & $\mathrm{x}$ & $-0,14928238204979$ \\
$\mathrm{c}$ & 0,724117730533323 & $\mathrm{E}$ & $-0,0765176967950394$ \\
\hline $\mathrm{R}^{2}$ & 0,998763 & $\mathrm{R}^{2}$ & 0,999397 \\
EAM & 1,589817 & $\mathrm{EAM}$ & 1,635327 \\
\hline
\end{tabular}

estimativas da intensidade máxima de precipitação diária. Para o município de Lavras - MG, o modelo exponencial foi o que melhor se ajustou aos dados estimados de intensidade de precipitação.

Adicionalmente, Borges e Thebaldi (2016) ao determinarem os parâmetros dos modelos de chuvas intensas IDF e exponencial para o município de Formiga MG, também encontraram melhor ajuste ao utilizar o modelo exponencial, diferentemente do observado em Divinópolis - MG.

\section{CONCLUSÃO}

A série histórica do município de Divinópolis MG, apresenta valores de precipitação máxima diária anual que variam de 35,1 mm (mínima) a 149,3 mm (máxima) para os anos estudados;

Apenas o modelo Fréchet não aderiu aos dados da série histórica, em ambos os testes de aderência realizados, as demais funções cumulativas de probabilidade tiveram aderência significativa tanto pelo teste KolmogorovSmirnov, quanto pelo Qui-Quadrado. Entretanto, o melhor ajuste se deu para o modelo Gumbel para Máximos, na qual 
no teste Qui-Quadrado apresentou o menor valor de $\lambda^{2}$ calculado, 2,025 aproximadamente.

Com relação às equações de chuvas intensas obtidas para Divinópolis - MG, o modelo IDF foi o que melhor se ajustou à série de intensidades máximas de precipitação, calculadas de acordo com a metodologia de desagregação de chuvas.

\section{REFERÊNCIAS}

ALVARES, C. A.; STAPE, J. L.; SENTELHAS, P. C.; GONÇALVES, J. L. M.; SPAROVEK, G. Köppen's climate classification map for Brazil. Meteorologische Zeitschrift, v.22, n.6, p.711-728, 2013. http://dx.doi.org/10.1127/0941-2948/2013/0507

ARAÚJO, L. E.; SOUSA, F. A. S.; RIBEIRO, M. A. F. M.; SANTOS, A. S.; MEDEIROS, P. C. Análise estatística de chuvas intensas na bacia hidrográfica do rio Paraíba. Revista Brasileira de Meteorologia, v.23, n.2, p.162-169, 2008.http://dx.doi.org/10.1590/S01027786200800020000 5

BORGES, G. M. R.; THEBALDI, M. S. Estimativa da precipitação máxima diária anual e equação de chuvas intensas para o município de Formiga, MG, Brasil. Ambiente \& Água, Taubaté, v. 1, n. 4, p. 891 - 902, 2016. http://dx.doi.org/10.4136/ambi-agua.1823

CARDOSO, C. O.; ULLMANN, M. N.; BERTOL, I. Análise de chuvas intensas a partir da desagregação das chuvas diárias de Lages e de Campos Novos (SC). Revista Brasileira de Ciência do Solo, v. 22, p. 131-140, 1998. http://dx.doi.org/10.1590/S0100- 06831998000100018

CRUCIANI, D. E.; MACHADO, R. E.; SENTELHAS, P. C. Modelos da distribuição temporal de chuvas intensas em Piracicaba, SP. Revista Brasileira de Engenharia Agrícola e Ambiental, v. 6, n. 1, p. 76-82, 2002. http://dx.doi.org/10.1590/S1415- 43662002000100014

FREITAS, A. J.; SILVA, D. D.; PRUSKI, F. F.; PINTO, F. A.; PEREIRA, S. B.; GOMES FILHO, R. R.; TEIXEIRA, A.F.; BAENA, L. G. N.; MELLO, L. T. A.; NOVAES, L. F. Equações de chuvas intensas no Estado de Minas Gerais. Belo Horizonte: Companhia de Saneamento de Minas Gerais; Viçosa: Universidade Federal de Viçosa, 2001. 65p.

FROEHLICH, D. C. Short-duration-rainfall intensity equations for drainage design. Journal of Irrigation and Drainage Engineering, v. 119, n. 5, p. 814-828, 1993. http://dx.doi.org/10.1061/(ASCE)07339437(1993)119:5(8 14)

HAAN, C.T. Statistical methods in hidrology. 2. ed. Ames: The Iowa State University Press, 1979. 377 p.
INSTITUTO BRASILEIRO DE GEOGRAFIA E ESTATÍSTICA - IBGE. Divinópolis - Dados gerais do município. Disponível em: http:/ / cidades.ibge.gov.br/painel/painel.php?lang=\&cod mun $=312230$ \&search $=\mid$ | infogr $\%$ E1 ficos:-dados-geraisdo-munic\%EDpio>. Acesso em: 09 de out. de 2016.

KOBIYAMA, M.; MENDONÇA, M.; MORENO, D. A.; MARCELINO I. P. V. A.; MARCELINO, E. V.; GONÇALVES, E. F.; BRAZETTI, L. L. P.; GOERL, R. F.; MOLLERI, G. S. F.; RUDORFF, F. M. Prevenção de desastres: conceitos básicos. Florianópolis: Ed. Organic Trading, 2006.

LANDSBERG, J. J. Some useful equations for biological studies. Experimental Agriculture, v.13, n.3, p.273-286, 1977. http://dx.doi.org/10.1017/S0014479700008000

MELLO, C. R.; FERREIRA, D. F.; SILVA, A. M.; LIMA, J. M. Análise de modelos matemáticos aplicados ao estudo de chuvas intensas. Revista Brasileira de Ciência do Solo, v. 25, p. 693-698, 2001.

http://dx.doi.org/10.1590/S010006832001000300018

MELLO, C. R.; SILVA, A. M. Hidrologia: Princípios e aplicações em sistemas agrícolas. Lavras: Ed. UFLA, 2013.

MELLO, C. R..; VIOLA, M. R. Mapeamento de chuvas intensas no estado de Minas Gerais. Revista Brasileira de Ciência do Solo, v.37, n.1, p.37-44, 2013. http://dx.doi.org/10.1590/S0100-06832013000100004

MELLO, C. R.; VIOLA, M. R.; MELLO, J. M.; SILVA, A. M. Continuidade espacial de chuvas intensas no estado de Minas Gerais. Ciência e Agrotecnologia. Lavras. v. 32. n. 2. p. 532-538, 2008. http://dx.doi.org/10.1590/S141370542008000200029

MORUZZI, R. B.; OLIVEIRA, S. C. Relação entre intensidade, duração e frequência de chuvas em Rio Claro, SP: métodos e aplicação. Teoria e Prática da Engenharia Civil, v.9, n.13, p.59-68, 2009.

NAGHETTINI, M.; PINTO, E. J. A. Hidrologia estatística. Belo Horizonte: CPRM, 2007. 552p.

OCCHIPINTI, A. G.; SANTOS, P. M. Relações entre as precipitações máximas de "um dia" e de " 24 horas" na cidade de São Paulo. São Paulo: Instituto Astronômico e Geofísico, 1966. 26p.

PINTO, F.A. Chuvas intensas no estado de Minas Gerais: análises e modelos. Viçosa: MG. Universidade Federal de Viçosa. p. 87. 1995. (Tese de Doutorado). 
RODRIGUES, J. O.; ANDRADE, E. M.; OLIVEIRA, T. S.; LOBATO, F. A. Equações de intensidade-duraçãofrequência de chuvas para as localidades de Fortaleza e Pentecoste, Ceará. Scientia Agraria, v.9, p.511-519, 2008. http://dx.doi.org/10.5380/rsa.v9i4.12505

SANSIGOLO, C. A. Distribuições de extremos de precipitação diária, temperatura máxima e mínima e velocidade do vento em Piracicaba, SP (1917-2006). Revista Brasileira de Meteorologia, v.23, n. 3, 2008, p.341-346. http:/ /dx.doi.org/10.1590/S0102-77862008000300009

SILVA, B. C.; CLARKE, R. T. Análise estatística de chuvas intensas na Bacia do Rio São Francisco. Revista Brasileira de Meteorologia. v.19. n.3, p.265-272. 2004.

SILVA, D. D.; PEREIRA, S. B.; PRUSKI, F. F.; GOMES FILHO, R. R.; LANA, A. M. Q.; BAENA, L. G. N. Equações de intensidade-duração-frequência da precipitação pluvial para o Estado de Tocantins. Engenharia na Agricultura, Viçosa, v. 11, n.1-4, p.7-14, 2003.

THEBALDI, M. S. Análise estatística da precipitação máxima diária anual da cidade de Uberaba e vazão mínima diária anual do Rio Uberaba. Revista Agrogeoambiental, v.4, n.3, 2012. http://dx.doi.org/10.18406/2316$1817 \mathrm{v} 4 \mathrm{n} 32012470$ 